\title{
O enfermeiro na prática do exame citopatológico de colo do útero: relato de experiência
}

\section{The nurse in the practice of cervix cytological examination: report of experience}

\author{
Simone Maria de Araújo Maia' • Carolliny Faria Jardim² • Claudia Cristina da Silva Moraes ${ }^{3}$ \\ Diogo Gomes Scotelario ${ }^{4}$ Gabriel Barreto da Silva Correia ${ }^{5}$ Lilian Maria dos Santos Silva $^{6}$
}

\begin{abstract}
RESUMO
O método principal e mais amplamente utilizado para deteç̧ão do câncer de colo do útero é o exame citopatológico ou exame Papanicolaou. Embora o exame tenha sido introduzido no Brasil há décadas, o câncer de colo uterino é considerado um problema de saúde pública. Vários fatores que podem interferir na prática de realização do exame, como a conduta dos profissionais que realizam a coleta do material. Objetiva-se ressaltar a importância do enfermeiro na coleta adequada do exame citopatológico do colo uterino antes, durante e depois da coleta. 0 estudo trata-se de um relato de experiência dos graduandos de enfermagem na prática do exame citopatológico. Com base no acompanhamento de trinta mulheres que compuseram ao estudo, a faixa etária das mesmas estava dentro do considerado grupo vulnerável no rastreamento do câncer cervical. Mesmo com o apoio estratégico do modelo de Saúde da Família, sabe-se que existem tabus da mulher relacionados à prática do exame. Diante disso, conseguiuse perceber a importância do acolhimento à mulher antes do exame e a aplicação dos cuidados de enfermagem. Concluí-se que o resultado desse estudo evidenciou a inserção do enfermeiro na contribuição para uma melhor prestação do serviço à saúde da mulher, relacionado à realização do exame citopatológico de forma adequada, na área da prevenção do câncer do colo de útero.
\end{abstract}

Palavras-chave: Teste de Papanicolaou; Cuidados de enfermagem; Saúde Pública.

\begin{abstract}
The primary and most widely used method for detecting cervical cancer is Pap smear or Pap smear. Although the test has been introduced in Brazil for decades, cervical cancer is considered a public health problem. Several factors that may interfere with the practice of conducting the exam, such as the conduct of professionals who perform the material collection. It is important to emphasize the importance of the nurse in the adequate collection of the cytopathological examination of the uterine cervix before, during and after collection. The study is an experience report of nursing undergraduates in the practice of cytopathological examination. Based on the follow-up of thirty women who composed the study, their age group was within the considered vulnerable group in cervical cancer screening. Even with the strategic support of the Family Health model, it is known that there are women's taboos related to the practice of the exam. Therefore, it was possible to perceive the importance of welcoming women before the examination and the application of nursing care. The results of this study evidenced the insertion of the nurse in the contribution to a better service delivery to women's health, related to the accomplishment of the cytopathological examination in an appropriate way, in the area of cervical cancer prevention.
\end{abstract}

Keywords: Cervical Smear; Nursing Care; Public Health.

${ }^{1}$ Especialista em Saúde Pública pela Universidade Gama Filho; Docente da Universidade Anhanguera Educacional - UNIAN Email: simoneamaia@yahoo.com.br

${ }^{2}$ Graduanda do $9^{\circ}$ período da Universidade Anhanguera Educacional - UNIAN. Email: carolliny_fj@hotmail.com

${ }^{3}$ Graduanda do $9{ }^{\circ}$ período da Universidade Anhanguera Educacional - UNIAN. Email: moraescristinam2016@gmail.com

${ }^{4}$ Graduando do $9{ }^{\circ}$ período da Universidade Anhanguera Educacional - UNIAN. Email: diogo_scott2@live.com

${ }^{5}$ Graduando do $9^{\circ}$ período da Universidade Anhanguera Educacional - UNIAN.Email: gabriielbarreto@hotmail.com

${ }^{6}$ Graduanda do $9{ }^{\circ}$ período da Universidade Anhanguera Educacional - UNIAN. Email: lilianscerda@hotmail.com 


\section{INTRODUÇÃO}

A incidência de câncer do colo do útero poder ser reduzida a partir de políticas que visem o monitoramento da doença, se baseando em sua ocorrência natural e no reconhecimento de que o câncer invasivo evolui a partir de lesões precursoras (lesões intraepiteliais escamosas de alto grau e adenocarcinoma in situ), que podem ser detectadas e tratadas adequadamente, impedindo a progressão da doença ${ }^{1-2}$. Segundo o Manual de Ministério da Saúde, o colo do útero é revestido por várias células epiteliais pavimentosas e de forma ordenada, que ao sofrerem alterações podem evoluir para câncer ${ }^{3}$.

O método principal e mais amplamente utilizado para detecção da doença é o exame Papanicolaou (exame citopatológico do colo do útero). Segundo a Organização Mundial da Saúde (OMS) com o mapeamento da população alvo e a garantia de diagnóstico e tratamento adequado dos casos, é possível reduzir em média, de 60 a $90 \%$ a incidência do câncer cervical invasivo ${ }^{4}$.

0 método de rastreamento do câncer do colo do útero no Brasil pelo exame citopatológico deve ser oferecido gratuitamente pelo Sistema Único de Saúde (SUS), com público-alvo destinado às mulheres de 25 a 64 anos e que já tiveram atividade sexual, com repetição do exame a cada três anos e após dois exames normais consecutivos realizados em intervalo de um $a n o^{5}$. Por outro lado, não é recomendado o rastreamento de mulheres sem história de atividade sexual ou submetidas à histerectomia total por outras razões que não o câncer do colo do útero ${ }^{6}$. Embora o exame citopatológico tenha sido introduzido no Brasil desde a década de 50 , a doença é considerada um problema de saúde pública.

O Sistema de Informação do Câncer do Colo do Útero (SISCOLO) foi desenvolvido pelo INCA em 1999, em parceria com o Departamento de Informática do SUS, como ferramenta para gerenciar as ações do programa de controle do câncer de colo do útero ${ }^{7}$. 0 sistema está implantado nos laboratórios que realizam o exame citopatológico (módulo do prestador de serviço) e nas coordenações estaduais, regionais e municipais de detecção precoce do câncer (módulo de coordenação). Atualmente, existe o Sistema de Informação do Câncer (SISCAN) que integra o SISCOLO e o SISMAMA ${ }^{7}$.

O exame preventivo do câncer do colo do útero (Papanicolaou) é a principal estratégia para detectar lesões precursoras e diagnosticar a doença. 0 exame pode ser feito em postos ou unidades de saúde da rede pública que tenham profissionais capacitados. 0 exame preventivo é indolor, simples e rápido. Percebe-se um desconforto que diminui se a paciente conseguir relaxar. Contudo, é necessário a utilização da técnica correta. Além de servir para a detecção de lesões precursoras do câncer do colo do útero o exame promove a detecção de infecção pelo Vírus Papiloma Humano (do inglês Human Papilloma Virus - HPV) ou outras infecções do gênero8.
O exame citopatológico está suscetível a vários fatores que podem interferir no seu aproveitamento, sendo o principal deles, relacionado aos profissionais que realizam a coleta do material. A coleta deve ser realizada por enfermeiros ou médicos, porém, o auxiliar e o técnico de enfermagem participam do procedimento desde a orientação e preparo da paciente para o exame, quanto no preparo da sala de exames, cuidados para o correto acondicionamento e transporte do material biológico, até que o mesmo seja enviado ao laboratório analítico?.

A resolução COFEN n 385/2011 apresenta a coleta do Papanicolaou como prática de cuidado complexo e que precisa ter conhecimento científico, sendo o profissional enfermeiro capacitado para realização do exame. E aplicando a partir da queixa uma prescrição de cuidados de enfermagem ${ }^{10}$.

Portanto, considerando que o profissional enfermeiro faz parte da equipe de profissionais que realizam a coleta do exame citopatológico, faz-se necessário a orientação dos enfermeiros a fim de mapear possíveis fatores que possam interferir no sucesso do exame ${ }^{11}$. A concentração de esforços governamentais aliada à produção acadêmica e à atuação efetiva dos profissionais da área pode trazer maiores avanços na prática do exame e contribuir para melhor atendimento à população feminina.

O objetivo do trabalho é ressaltar a importância do enfermeiro na coleta adequada do exame citopatológico do colo uterino, antes, durante e depois da coleta. A relevância das ações desenvolvidas pelo enfermeiro no exercício da prevenção e a promoção da saúde se delineou o objeto deste estudo. A problemática se direcionou a importância desse profissional quanto à realização do exame citopatológico e consequente melhoria da qualidade de vida das pacientes ${ }^{10}$. Este estudo advém da vivência dos autores no Estágio Supervisionado em Saúde Coletiva como acadêmicos do curso superior de enfermagem, na contribuição para aprendizagem acadêmica e novas pesquisas.

\section{MÉTODO}

O estudo trata-se de um relato de experiência dos graduandos de enfermagem na prática do exame citopatológico. 0 universo do estudo foi composto pelo total de trinta mulheres atendidas no período entre 3 de maio a 29 de junho de 2016, em uma instituição do Sistema Único de Saúde. A percepção deu-se através do antes e o depois da realização do exame.

0 estudo teve como questão norteadora a coleta de exame citopatológico pelos graduandos em enfermagem como parte do Estágio Supervisionado em Saúde Coletiva, o qual faz parte de uma instituição de ensino superior localizada na cidade de Niterói, Rio de Janeiro. As atividades foram supervisionadas pela docente responsável no campo do estágio e acompanhadas pela equipe de enfermagem da unidade de saúde contudo, em outro município. 


\section{RESULTADOS E DISCUSSÃO}

Com base no acompanhamento de trinta mulheres que compuseram ao estudo, a faixa etária das mesmas estava dentro do considerado grupo vulnerável no rastreamento do câncer cervical.

Com aproximadamente 500 mil novos casos por ano no mundo, o câncer do colo do útero está classificado como o segundo tipo de câncer mais comum entre mulheres, perdendo apenas para o câncer de mama ${ }^{12}$. No Brasil, para esse tipo de câncer é constatado em média 20 mil casos anuais. Ainda no Brasil, existem cerca de seis milhões de mulheres entre 35 a 49 anos de idade que nunca realizaram o exame citopatológico do colo do útero (Papanicolaou) 9 . Como consequência surge milhares de novos casos da doença anualmente. A detecção precoce desta neoplasia ou das lesões precursoras é muito importante já que a cura pode chegar a $100 \%$ e na maioria dos casos a detecção ocorre ainda em nível ambulatorial ${ }^{13}$.

A Saúde da Família é um modelo de serviço que está no primeiro nível de atenção no SUS e é considerada uma estratégia primordial para a organização e o fortalecimento da atenção básica. A estratégia da Saúde da Família é apresentar vínculo com a comunidade e ações dentro dos níveis da assistência, identificando fatores de risco e intervindo de forma adequada. Humaniza as práticas de saúde, busca a satisfação dos usuários por meio do estreito relacionamento dos profissionais com a comunidade e sempre correlaciona à saúde como direito de cidadania ${ }^{11}$.

Com relação à prevenção do câncer do colo do útero, esse modelo tem sido primordial no acompanhamento em nível de atenção primária à saúde, voltadas para a melhoria da cobertura do exame. Estas incluem esclarecer e informar à população feminina sobre o rastreamento, identificar aquelas que pertencem à faixa etária prioritária e/ou grupos considerados de risco, captar e realizar a coleta de citologia, detectar e sensibilizar as que se ausentaram. No entanto, estudos apontam que não se deve esperar apenas a presença voluntária das mulheres para a realização do exame, mas é necessário programar formas de recrutamento por meio de ações educativas e triagem, em que a organização da atenção à saúde nos moldes da Saúde da Família favorece a criação desse vínculo ${ }^{14}$.

Estudos mostram que rastrear mulheres muito jovens não tem impacto na redução da incidência desse tipo de câncer, visto que, nesse grupo populacional as lesões predominantes são de baixo grau e as mesmas regridem espontaneamente entre seis e dezoito meses ${ }^{5}$. A faixa etária mais propensa à fatalidade pelo câncer de colo do útero é entre 50 e 60 anos $^{5}$. A precocidade das relações sexuais e da gravidez são fatores de risco para o câncer cervical, talvez porque, na adolescência, a metaplasia se intensifica, e o coito aumenta a probabilidade de transformação atípica ${ }^{15}$.

0 procedimento de coleta do material se dá pelo preparo prévio da paciente, seguido da inserção do espéculo através do óstio vaginal para visualização do colo uterino. As células podem, portanto, ser colhidas do fundo de saco posterior da vagina (conhecido como fundo de saco vaginal ou fundo de saco de Douglas), do ectocérvice (abertura do colo) e também, da endocérvice (interior do colo). 0 conteúdo é então fixado sobre a lâmina imediatamente. 0 material retirado de cada área do colo deve ser espalhado na lâmina em direções diferentes para que haja, ao final da coleta, duas regiões distintas ${ }^{16}$.

A mulher, para colher o exame não deve estar durante - seu ciclo menstrual, usar duchas ou medicamentos vaginais por 48 horas antes do exame (pois ficará com toda a flora bacteriana alterada e também o epitélio celular local, prejudicando a análise e o resultado obtido), ter contato sexual 24 horas antes do exame, as gestantes só podem realizar o exame após o terceiro mês de gestação, pelo risco da coleta da endocérvice, podendo desencadear alguma dilatação ou sangramento?.

Mesmo com todas essas estratégias para a realização do exame citopatológico, sabe-se que existem com o processo muitas dificuldades relacionadas à adesão das mulheres. O exame é considerado por muitas, um procedimento invasivo, que gera medo, vergonha, ansiedade, desconforto e repulsa da própria genitália, gerando prolongados adiamentos na procura do serviço de saúde.

Pensando nisso, o estudo em questão, analisou a importância da coleta do material e realização do exame, enfatizando aos autores submetidos à prática do estágio, a postura técnica e ética no sentindo de preservar a privacidade da paciente, posicioná-la em uma posição confortável, além de ser capaz de explicar os procedimentos realizados, observando sempre se a mesma compreendeu as explicações. A tabela 1 relaciona as dificuldades na adesão das mulheres ao exame Papanicolaou, segundo o relato, com os cuidados prescritos pelos acadêmicos de enfermagem para cada problema percebido. O campo prático para estágio e realização da pesquisa foi no município de Rio Bonito, Rio de Janeiro, 2016.

Durante o cuidado de enfermagem, as pacientes demonstraram preocupação questionando a interferência de fatores relacionados à falta de conhecimento sobre o exame e o despreparo dos profissionais no momento da coleta do material. Assim conseguiu-se perceber a importância de dois tópicos: 0 acolhimento à mulher antes do exame e a aplicação dos cuidados de enfermagem.

A imagem do enfermeiro torna-se crucial nesta etapa. O profissional deve reconhecer que o medo do câncer é um obstáculo na procura da assistência e deve estar preparado para atuar na dimensão do cuidar, prevenindo e detectando precocemente qualquer suspeita da doença. A mulher tem acesso aos serviços do sistema único de saúde e busca ter a resposta às suas necessidades. Antes da realização do exame, os autores explicaram o que é o exame e a técnica da coleta, utilizando a linguagem coloquial e não técnica sobre o assunto, além de parabenizá-las por se propor ao cuidado com a saúde. Com responsabilidade e compromisso de prestar assistência integral à população na unidade de 
Tabela 1. Percepção dos graduandos de enfermagem na realização do exame citopatológico, a partir da experiência em um centro de saúde na cidade de Rio Bonito, Rio de Janeiro

\begin{tabular}{l|c}
\hline \multicolumn{1}{c|}{$\begin{array}{c}\text { Diagnóstico de } \\
\text { Enfermagem }\end{array}$} & Prescrição de Cuidados de Enfermagem \\
\hline $\begin{array}{l}\text { Relação sexual } \\
\text { prejudicada }\end{array}$ & $\begin{array}{c}\text { Orientar para a mudança no padrão da } \\
\text { sexualidade; Oferecer apoio à usuária e } \\
\text { ao companheiro. }\end{array}$ \\
\hline Ansiedade & Estabelecer relação de confiança. \\
\hline Angustia & $\begin{array}{c}\text { Retirar as dúvidas antes de proceder à } \\
\text { coleta do exame. }\end{array}$ \\
\hline $\begin{array}{l}\text { Déficit de } \\
\text { autocuidado }\end{array}$ & $\begin{array}{c}\text { Estimular a usuária na participação das } \\
\text { atividades da vida diária. }\end{array}$ \\
\hline $\begin{array}{l}\text { Padrão de } \\
\text { eliminação } \\
\text { prejudicada }\end{array}$ & $\begin{array}{c}\text { Manter higiene íntima; Avaliar } \\
\text { eliminações vesico-intestinais. }\end{array}$ \\
\hline $\begin{array}{l}\text { Aceitação do } \\
\text { estado de saúde }\end{array}$ & $\begin{array}{c}\text { Oferecer informações sobre diagnóstico, } \\
\text { tratamento e prognóstico. }\end{array}$ \\
\hline $\begin{array}{l}\text { Risco de } \\
\text { sangramento }\end{array}$ & $\begin{array}{c}\text { Observar e informar a enfermeira } \\
\text { sangramento após relação sexual. }\end{array}$ \\
\hline \begin{tabular}{l} 
Risco de infecção \\
\hline $\begin{array}{l}\text { Fonte: Diagnósticos de enfermagem segundo a North American Nursing } \\
\text { Diagnosis Association (Nanda) }\end{array}$
\end{tabular}
\end{tabular}

saúde, ouviu-se as queixas, através de uma escuta ativa e aplicando a conduta necessária.

Também pode-se perceber que com a atuação do enfermeiro na educação em saúde, no incentivo à realização anual do exame Papanicolaou, nas conversas onde são esclarecidos mitos e medos relacionados ao exame preventivo, na busca ativa desta população e na realização do próprio exame, os índices de mulheres que se previnem podem aumentar a cada ano, e os índices de novos casos diminuírem consideravelmente ${ }^{18}$.

Durante a consulta o enfermeiro deve realizar uma completa anamnese, preparar a paciente para o exame, realizar a técnica da coleta propriamente dita, ser capaz de perceber intercorrências, observar as necessidades de se realizar encaminhamentos e ao final da consulta enfatizar a importância do retorno em tempo adequado. Durante a realização do exame é necessário criar um vínculo com a paciente para que a consulta se torne mais humanizada e para que a cliente se sinta mais a vontade ${ }^{10}$.

O enfermeiro precisa trabalhar com os fatores negativos do exame preventivo, que trava algumas das mulheres a realizar o mesmo. Já para outras, o exame é visto como um fator positivo focado para o autocuidado com seu corpo e faz com que elas reconheçam a importância da prevenção e detecção precoce do câncer de colo de útero e ajudando em ter uma vida saudável. É esse profissional quem irá organizar a assistência desenvolvendo métodos estratégicos e criativos para a realização do rastreamento das usuárias do Centro de Saúde, incentivando-as a realizarem o exame periódico. Ainda de acordo com os autores, é de extrema importância que o enfermeiro exponha a paciente as técnicas utilizadas nos exames, forneça informações para o momento da coleta e crie espaços de privacidade durante a consulta.

Neste contexto, percebe-se a necessidade do enfermeiro em trabalhar a educação em saúde e quebrar tabus a respeito do exame citopatológico. Faz-se necessário ainda, sinalizar a importância da realização desse procedimento, retratar sua eficácia na prevenção e detecção precoce do câncer do colo do útero para a mulher que o realiza com regularidade, sempre com vistas a atingir a população de maior risco.

\section{CONCLUSÃO}

O resultado desse estudo evidenciou a inserção do enfermeiro na contribuição para uma melhor prestação do serviço à saúde da mulher, relacionado à realização do exame citopatológico de forma adequada, na área da prevenção do câncer do colo de útero. É primordial a percepção antes, durante e depois na assistência à essa mulher. Diante disso, observou-se a importância do papel do enfermeiro para com essa população, levando as pacientes maior esclarecimento, conforto, confiança na prevenção desta patologia e empoderando-a no seu próprio cuidado. Deixando claro que o exame deve ser realizado mesmo na ausência de problemas ginecológicos.

\section{REFERÊNCIAS}

1. BRASIL. INCA. Colo do útero: detecção precoce [Internet] 2011 [acesso em 07 de julho 2016]. Disponível em: http:// www2.inca.gov.br/wps/wcm/connect/tiposdecancer/site/ home/colo_utero/deteccao_pre coce.

2. Soost HJ, Lange HJ, Lehmacher W, Ruffing-Krellmann B. The validation of cervical cytology: sensitivity, specificity and predictive values. Acta Cytol [Internet]. 1991 [acesso em 03 de maio 2016]; 35(1):8-13. Disponível em: https://www. researchgate.net/publication/21160652_The_validation_of_ cervical_cytology_Sensitivity_specificity_and_predictive_value.

3. Brasil. Ministério da Saúde. Secretaria de Atenção à Saúde. Instituto Nacional de Câncer. Coordenação de Prevenção e Vigilância de Câncer. Estimativas 2008: incidência de câncer no Brasil. 2007.

4. Kerkar RA, Kulkarni YV. Screening for cervical cancer: An overview. J Obstet Gynecol India [Internet]. 2006 [acesso em 15 de junho 2016]; 56(1):115-122. Disponível em: http:// medind.nic.in/jaq/t06/i2/jaqt06i2p115.pdf.

5. Ministério da Saúde. Datasus. Informações de Saúde. Câncer do colo do útero. Disponível em: http://w3.datasus.gov. br/siscam/index.php?area=01. 2008 [acesso em 06 de maio 2016].

6. Instituto Nacional de Câncer (Brasil). Diretrizes Brasileiras para o Rastreamento do Câncer do Colo do Útero. Disponível em: http://www1.inca.gov.br/inca/Arquivos/Titulos/Nomenclatura_colo_do_utero.pdf. 2011 [acesso em 06 de julho 2016].

7. Brasil. Ministério da Saúde. Secretaria de Atenção à Saúde. Departamento de Atenção Básica. Rastreamento (Série A: 
Normas e Manuais Técnicos. Cadernos de Atenção Primária n²9). Brasília; 2010, p. 41-89.

8. Pinho AA, França Júnior I. Prevenção do câncer de colo do útero: um modelo teórico para analisar o acesso e a utilização do teste de Papanicolaou. Rev Bras Saude Mater Infant [Internet]. 2003 [acesso em 22 de maio 2016]; 3(1):3485. Disponível em: http://www.scielo.br/scielo.php?pid=S15 1938292003000100012 \&script=sci_abstract \&tlng=pt.

9. Instituto Nacional do Câncer (INCA). Periodicidade de realização do exame preventivo do câncer de colo do útero: normas e recomendações do Instituto Nacional do Câncer. Rev Bras Cancerol [Internet]. 2002 [acesso em 17 de maio 2016]; 12(4):508-515. Disponível em: http://www1.inca.gov. $\mathrm{br} / \mathrm{rbc} / \mathrm{n}_{-} 48 / \mathrm{v} 01 / \mathrm{pdf} /$ normas.pdf.

10. Brasil. Resolução COFEN N 385/2011. Normatiza a execução, pelo Enfermeiro, da coleta de material para colpocitologia oncótica pelo método de Papanicolaou. Disponível em http://www. cofen.gov.br/resoluo-cofen-n-3852011_7934. html. 2011 [acesso em 06 de maio 2016].

11. Marçal JA, Gomes LTS. A prevenção do câncer de colo de útero realizada pelo enfermeiro na Estratégia Saúde da Família: Revisão integrativa da literatura. REAS [Internet]. 2013 [acesso em 20 de junho 2016]; 5(2):474-489. Disponível em: http:// acervosaud.dominiotemporario.com/doc/artigo_035.pdf.

12. World Health Organization. International Agency for Research on Cancer. Globocan [Internet].2011. [acesso em 10 de maio 2016]. Disponível em: http://globocan.iarc.fr/.

13. Santos MCL, Fernandes AFC, Cavalcante PP. Consulta ginecológica: motivações e conhecimento da mulher sobre a prevenção do câncer do colo do útero. Rev RENE [Internet]. 2004 [acesso em 01 de junho 2016]; 5(1):22-26. Disponível em: www.revistarene.ufc.br/revista/index.php/revista/article/ download/842/pdf.

14. Mistura C, Mistura C, Silva RCC, Sales JRP, Melo MCP, Sarmento SS. Papel do enfermeiro na prevenção do câncer de colo uterino na estratégia saúde da família. Revista Contexto \& Saúde [Internet]. 2011 [acesso em 23 de junho 2016]; 10(20):1161-1164. Disponível em: https://www.revistas.unijui.edu.br/index.php/contextoesaude/article/view/1763.

15. Fernandes MS, Fonseca RLS, Silva BPM, Barbosa FKS, Almeida IRC. Papel da Enfermagem na Prevenção de Infecção por HPV em adolescente e jovens. In: Resumos do I Congresso Nacional de Ciências da Saúde; 2014; Cajazeiras; 2014. p. 28.

16. Santos ML, Moreno MS, Pereira VM. Exame de Papanicolaou: qualidade do esfregaço realizado por alunos de enfermagem. Rev bras cancerol. [Internet]. 2009 [acesso em 02 de junho 2016]; 55(1):19-25. Disponível em: http://www.inca.gov.br/rbc/n_55/v01/pdf/05_artigo_exame_Papanicolaou.pdf.

17. Braga CG, da Cruz DALM. A Taxonomia II proposta pela North American Nursing Diagnosis Association (NANDA). Rev Latino-am Enfermagem. 2003 [acesso em 30 de maio 2016]; 11(2):240-245. Disponível em: http://www.scielo.br/pdf/ rlae/v11n2/v11n2a16.pdf.

18. Melo MCSC, Vilela F, Salimena AMO, Souza, IEO. O Enfermeiro na Prevenção do Câncer do Colo do Útero: o Cotidiano da Atenção Primária. Rev Bras Cancerol [Internet]. 2012 [acesso em 01 de maio 2016]; 58(3): 389-398. Disponível em: http://www1. inca.gov.br/rbc/n_58/v03/pdf/08_artigo_enfermeiro_prevencao_cancer_colo_utero_cotidiano_atencao_primaria.pdf. 